\title{
Mobile Music Touch: Vibration Stimulus in Hand Rehabilitation
}

\author{
T. Markow, N. Ramakrishnan, K. Huang, T. Starner, \\ M. Eicholtz, S. Garrett, H. Profita, A. Scarlata, \\ C. Schooler, A. Tarun \\ College of Computing \\ Georgia Institute of Technology \\ Atlanta, Georgia, 30332 United States \\ \{tmarkow3 | narayanan911 | kevinhh | thad | m.eicholtz | \\ garrett | hprofita | ascarlata3 | chip.schooler | aneesh \} [at] \\ gatech.edu
}

\author{
D. Backus \\ Crawford Research Institute \\ Shepherd Center \\ Atlanta, Georgia, 30309 United States \\ deborah_backus [at] shepherd.org
}

\begin{abstract}
Hand rehabilitation often consists of repetitive exercises, which may result in reduced patient compliance and decreased results. The Mobile Music Touch (MMT) is proposed as an engaging form of hand rehabilitation. MMT is a lightweight, wireless haptic music instruction system consisting of gloves and a mobile Bluetooth-enabled computing device, such as a mobile phone. Musical passages to be learned via "passive haptic learning" are loaded into the mobile device and played repeatedly while the user performs other tasks. As each note of the music plays, vibrators on each finger in the gloves activate, indicating which finger to use to play each note. We present observations from a pilot study of MMT used for hand rehabilitation for people with tetraplegia resulting from incomplete Spinal Cord Injury (SCI); observations from a study conducted on able-bodied people, providing baseline data for assessment methods; and observations on glove design for persons with tetraplegia.
\end{abstract}

Keywords- Tactile, Haptic, Rehabilitation, Spinal Cord Injury, Wearable

\section{MOTIVATION AND RELATED WORK}

Rehabilitation is a valuable tool that can help people with SCI regain some, and perhaps all, of the use of their hands and arms. It has been documented that motivation is a prime factor in the recovery process [5]. Unfortunately, many traditional therapies involve monotonous repetitions of certain motions every day. For example, people undergoing hand rehabilitation may be asked to touch their thumb to their fingers on the same hand or squeeze a ball for several hours a day. These routines are sometimes quickly abandoned. On the other hand, it has been shown that playing instruments such as the keyboard can improve finger strength and dexterity as well as feeling of mental well-being [8]. Also, it has been shown that stimulation of the afferent (sensory) nerves can increase flexibility and range of movement and decrease muscle spasticity [6]. Dimitrejevic writes that "diminished afferent input to the brain from the affected hand is a common deficit after stroke. People with SCI may become less aware of their affected upper extremity because of sensory loss and partial

Permission to make digital or hard copies of all or part of this work for personal or classroom use is granted without fee provided that copies are not made or distributed for profit or commercial advantage and that copies bear this notice and the full citation on the first page. To copy otherwise, to republish, to post on servers or to redistribute to lists, requires prior specific permission and/or a fee.

PERVASIVEHEALTH 2010, March 22-25, Munchen, Germany

Copyright $\odot 2010$ ICST 978-963-9799-89-9

DOI 10.4108/pervasivehealth.2010.8 paralysis. As a consequence, they use that extremity less and less, learning to use the unaffected arm in its place. Over time, disuse weakens muscles and most likely reduces the representation area of the affected part in the cortex" [6]. Doidge [7] discusses similar mechanisms for many different aspects of rehabilitation.

MMT is a lightweight, wireless haptic music instruction system comprised of a glove and a mobile Bluetooth-enabled computing device, such as a mobile phone [3]. This system was used in a previous study to teach users to play simple songs passively while engaged by a separate, distracting task, for example, completing a reading comprehension test. The subjects who received the vibration stimuli with the MMT system performed better than the control group that had to play the song without the vibration input during practice (Fig 1) [3].

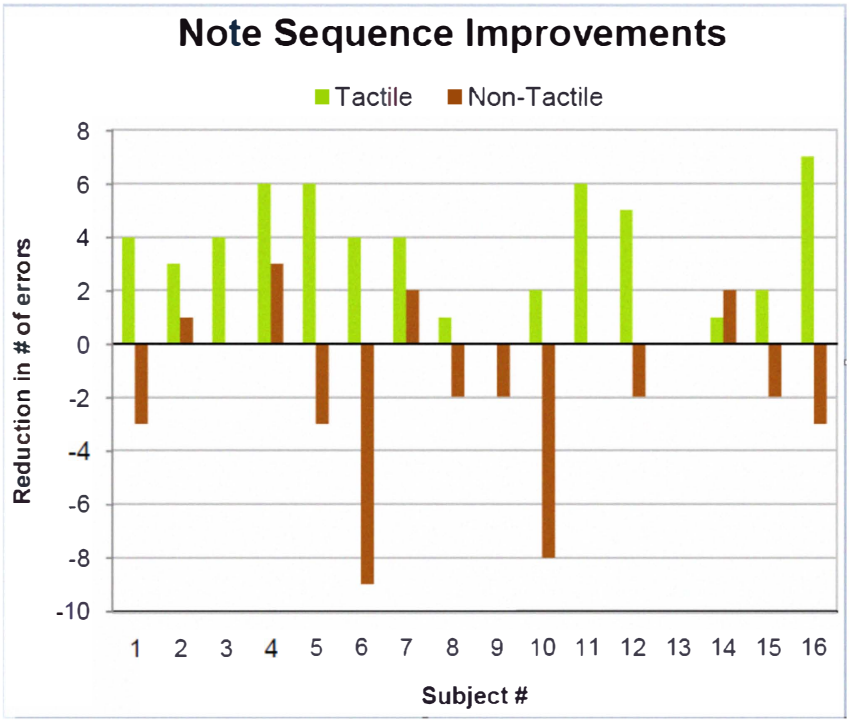

Figure 1. Participants using MMT, provided with tactile stimulus versus those who simply performed typical piano practice. This shows that those who received tactile stimulus had a greater reduction in errors when playing back learned songs than those without the tactile input. 


\section{MMT REHABILITATION}

In discussing MMT with members of the staff at the Shepherd Center, which specializes in SCI, it was thought that perhaps the MMT could provide a way for the SCI user to improve dexterity and sensation while learning a new skill set. Based on this discussion, we designed an MMT pilot study with two people with SCI; this pilot study demonstrated that the system has potential as a form of hand rehabilitation [3]. MMT can stimulate patients' afferent nerves and help them exercise their hands in a fun way while teaching them a lifelong skill to promote continued exercise in the long term. Aside from sensory function, MMT has the potential to help improve motor function [3]. MMT may be able to provide the tactile stimulation needed to diminish the extent of deficit from de-afferentiation of the hand. The original MMT system consists of a wireless glove with vibration motors in each finger and an electronic piano keyboard connected to a laptop via a USB connection. The piano keyboard includes Light Emitting Diodes (LEDs) under each key that can be lit under program control (Fig. 2).

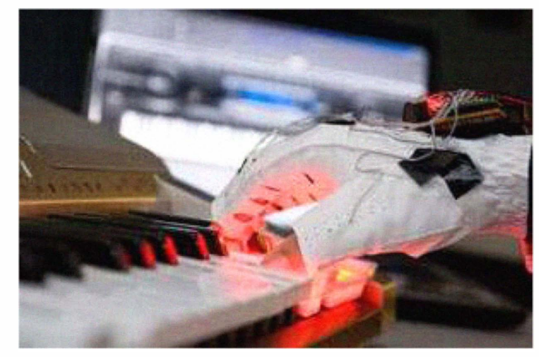

Figure 2. Mobile Music Touch "active practice" system.

Our software sends data to the piano keyboard to specify which key to light and to the MMT glove over a Bluetooth connection to indicate which finger should vibrate (i.e. which is the correct finger that is used to press the key). The pilot study consisted of three 30 minute "active practice" sessions a week over a period of four weeks. Using the system in the original "active practice" mode, where the participant plays simple songs with auditory, haptic (via vibration motors in the glove), and visual cues (provided by LEDs built into the keyboard) provided a form of potential rehabilitation in a fun and engaging way. Both participants in the pilot study stated subjectively that they enjoyed the sessions and perceived improvements in the ability to use their hands. In both cases, there were also noted improvements in the Semmes Weinstein monofilament evaluation as well as on the Grasp and Release Test (GRT), which were used to assess sensory perception in the hands. On the GRT, increases in the number of repetitions shows improvement, on the Semmes Weinstein test, a decrease demonstrates positive change, as this means the participant is able to feel a smaller diameter filament. Data collected with these methods on the participants is quantified in Figures 3-5.

\section{GRT Results SCI Pilot Study}

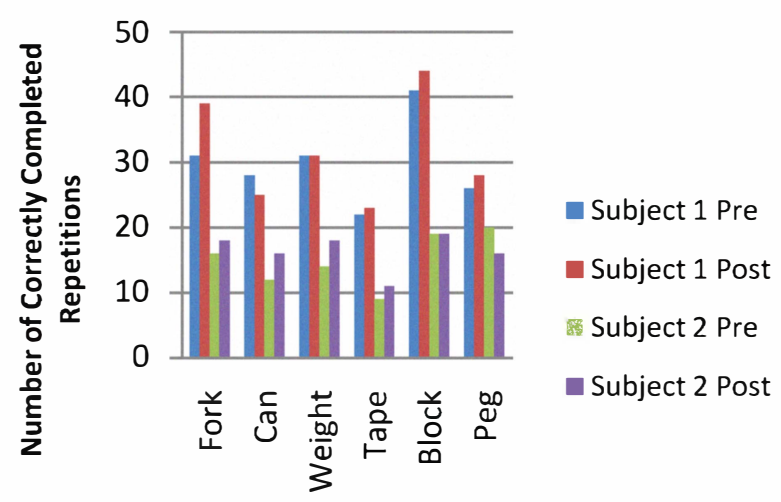

Events

Figure 3. Grasp and Release Test (GRT) results of Spinal Cord Injury Pilot Study.

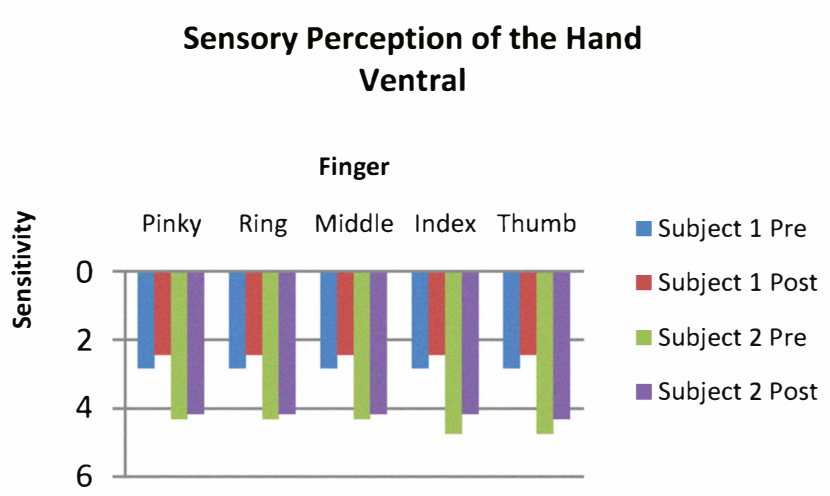

Figure 4. Semmes Weinstein monofilament evaluation data, Sensory Perception of the Hand, Ventral

\section{Sensory Perception of the Hand Dorsal}

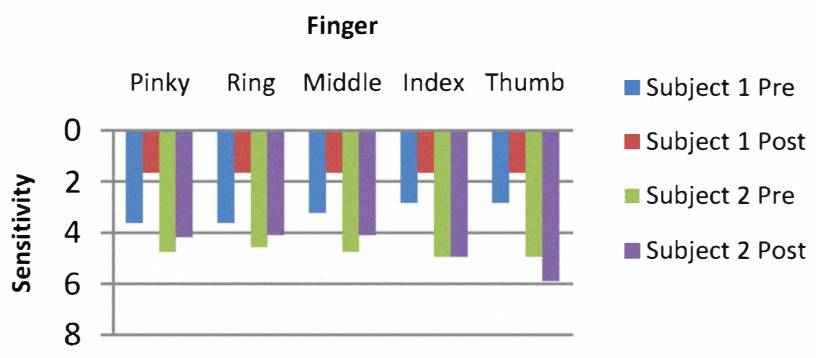

Figure 5. Semmes Weinstein monofilament evaluation data, Sensory Perception of the Hand, Dorsal

Both participants had reached a plateau in their hand rehabilitation, prior to this study, for a period of at least eight months. This pilot study demonstrated the potential for MMT as a possible form of rehabilitation in cases of tetraplegia 
resulting from SCI. The pilot study also raised some questions and issues. We realized that we would require a new, more durable glove than the original prototype, which could not withstand the abuse of more than a handful of subjects. In order to verify the rehabilitative effect on a larger SCI population and isolate the cause, we would need a new glove system capable of operating independently.

Because of the nature of the pilot study design, it was not possible to determine if the cause of the improvements could be attributed to the vibration stimulus, the act of playing the piano, the music, or a combination of any or all of these influences. The population of persons with incomplete Spinal Cord Injury who meet the criterion for this study is quite small, and it is difficult to gain access to the group. Thus, we recognized the need to collect able-bodied baseline data for the assessments commonly used in hand rehabilitation, including the Grasp and Release Test (GRT) [1], and tests of sensory perception in the hands, specifically the 2-Point Discrimination Test [4]. We also constructed an Action Research Arm Test (ARAT) kit [2]. Having never conducted this particular test, we needed to refine administering it on able-bodied people prior to using this evaluation method on people with SCI. Another issue we discovered was that of glove suitability for persons with SCI. In order to assess a larger group of persons with tetraplegia, we would need a glove that would be suitable for the wide variety of abilities and requirements that are found in this diverse population. Both the participants had trouble with the original glove, which consists of a golf glove with the finger tips cut off and vent holes cut into the palm (Fig. 6). A battery pack and electronic boards were mounted on the back of the wrist area, and wires were guided up the backs of the fingers to the vibration motors. This model was of acceptable size for the pilot study, as each participant happened to have large size hands, which was the size of the chosen golf glove; however, in addition to the potential sizing problem, this study revealed another issue - that of getting the glove on and off of the SCI subjects with "clawing" of the hands.

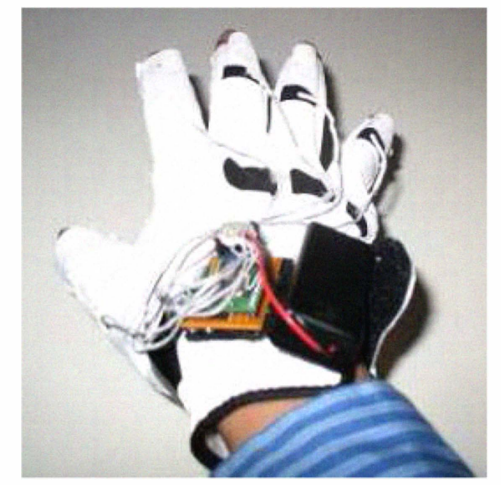

Figure 6. MMT glove used in the SCI pilot study.

In order to evaluate the full potential of the MMT as a form of rehabilitation, a full SCI "active practice" study must be conducted, with enough participants to validate the findings of the pilot study [3].

\section{MOBILE MMT SYSTEM}

Upon completion of the full SCI "active practice" study, we would need to isolate the cause (vibration, audio, or the "active practice" sessions on the piano keyboard) of any observed rehabilitative effects. In order to accomplish this, we need a system that will allow users to be tested in all of these ways. If it turns out that just the vibration and/or audio is causing the effect, then we will need a glove and hardware that one could imagine using throughout their normal day. For convenience, the glove should be controlled with a smaller device, preferably something our future users might already carry throughout the day. The "mobile" mode that was designed differs from the "active practice" system in that it allows the participant to wear the glove for long periods of time throughout a day, receiving vibration stimulus and/or music while performing normal daily activities. To this end, the software has been re-written in $\mathrm{C \#}$ and placed on a Windows Mobile cellular phone platform.

Given the MIDI file of the song to be learned, the cell phone software synthesizes the appropriate tones with simple sine wave synthesis through the use of Tone and SoundPlayer classes, freely available from the OpenNETCF organization to play the Musical Instrument Digital Interface (MIDI) format files of the songs. The software also provides the data to the glove via Bluetooth to command the vibration motors to fire in the correct finger for each note. An early version of the working "mobile" mode MMT glove and hardware is pictured in Fig. 7. The newly implemented GUI for the cellular phone based software is shown in Fig. 8 .

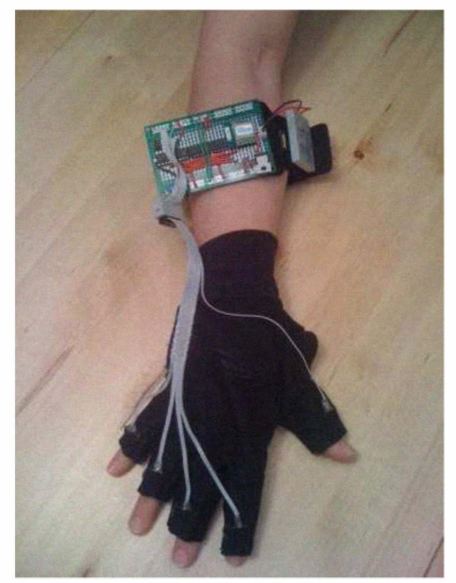

Figure 7. "Mobile" mode MMT glove and hardware. 


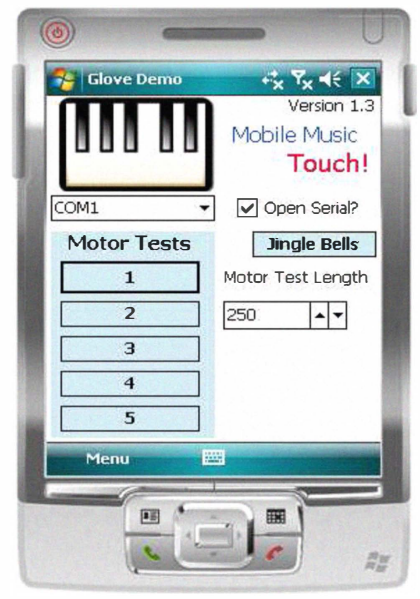

Figure 8. GUI for cellular phone based software for the "mobile" MMT system.

The Mobile Music Touch hardware is composed of 3 distinct parts: (1) actuators - small vibration motors, one for each finger, (2) a Bluetooth-to-serial module coupled with an ATMega328P chip to provide wireless connectivity, and (3) a computing device: a laptop computer in the case of the "active practice" system, or a cellular phone in the "mobile" system, which produces music output and controls the glove via Bluetooth to send synced vibrations.

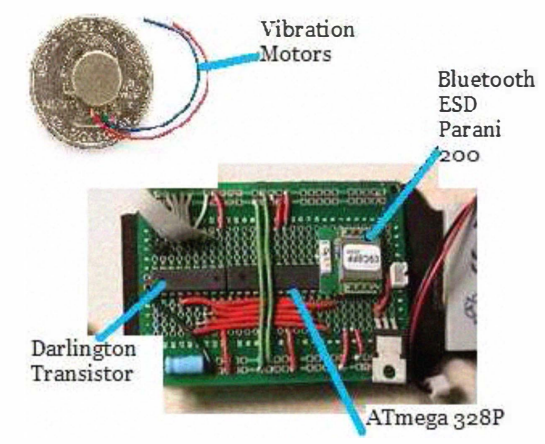

Figure 9. Hardware components for new MMT system

Fig. 9 shows the primary components used for the hardware. The Bluetooth module is a Bluetooth-to-serial DIP module from Sparkfun Electronics. The ATmega 328P was programmed using $C$ to power on the ESD Parani 200 Bluetooth module using the AT commands. The new MMT system sets the Bluetooth module to a mode where it can scan for possible connections. If a connection is found the laptop sends Bluetooth information to the module, which is then replicated on its serial pin. The serial output is then fed into the RX pin of the ATMega328p chip. The ATMega328p chip was programmed to read the serial information and output current on its pins to drive the motors accordingly. Instead of individual wires, ribbon cable was employed to connect the motors to the board in order to promote better wire management. The ribbon cable splits into individual wires near the knuckle of each finger, and vibration the motors were surface soldered to the single wires. In addition, a portion of the ribbon cable was sewn onto the glove near the wrist to further prevent snagging of the wires. The Bluetooth receiver, controller, and rechargeable Lithium polymer battery was housed in one box (Fig. 10). The housing was not created in time for deployment in the field study, but it is a viable option for future studies. The complete system is shown in Fig. 7.

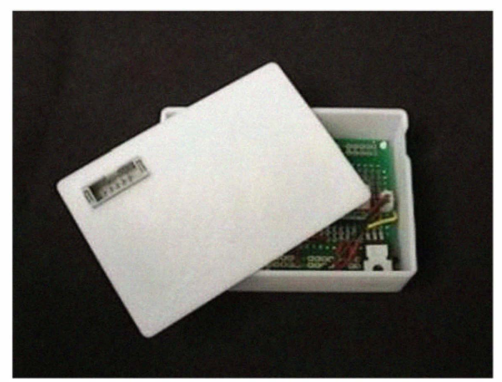

Figure 10. Custom housing compartment for hardware.

\section{ABle-Bodied Study of Assessment Methods}

Our target population, persons with spinal cord injury, is not very large; there are only approximately 250,000 people in the United States who fit this category [9]. As a result, the available number of participants is small. We decided to conduct an able-bodied experiment using the "active practice" system that was used during the pilot study, in order to collect able-bodied data for the assessment methods used to evaluate persons with SCI. This data provided us with a baseline from which to measure any potential change as a result of using the MMT system. We also sought to refine our administration of the evaluations on able-bodied persons to ensure our own smooth performance during the full SCI study with injured persons. The able-bodied study was executed over a period of two weeks, with three 30 minute sessions per week. The participants were broken down into two groups, the experiment group of five people and a control group of four. Both groups underwent three forms of pre and post assessment used to assess persons with tetraplegia. Specifically the Grasp and Release Test (GRT) [1] and the Action Reach Arm Test (ARAT) [2], were used to test for upper extremity motor skills. The 2-Point Discrimination Test [4] was used to determine sensory perception in the hands. The ARAT is designed specifically for those who have suffered incomplete spinal cord injury [2], and thus a ceiling effect was encountered with all the able-bodied participants.

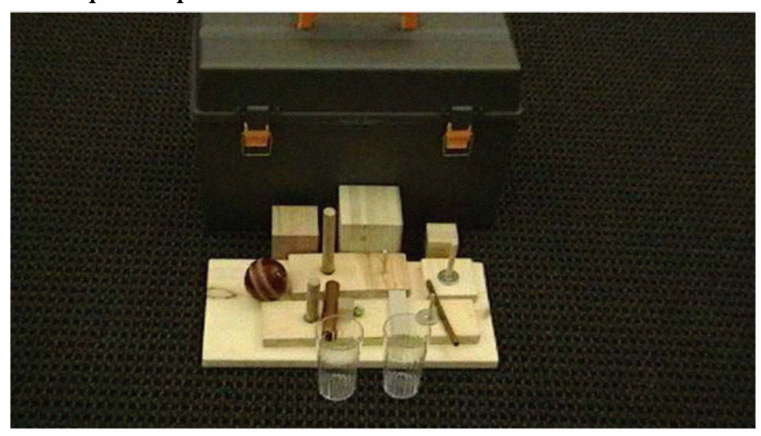

Figure 11. Action Research Arm Test (ARAT) kit. 
The Grasp and Release Test (GRT) consists of several repetitive activities that evaluate a person's ability to employ a set of common hand grasp positions used in normal daily activities. These grasps are evaluated using the following subtests: fork, can, weight, tape, block, and peg. The baseline able-bodied data is presented in Figures 12-17. In each graph, subjects 1-5 were in the experimental group, while 6-9 comprised the control group.
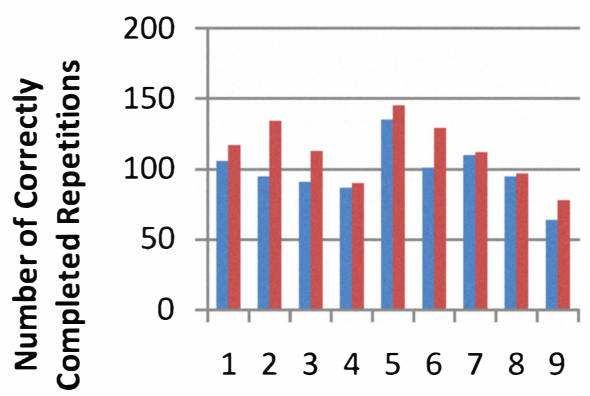

$\begin{array}{lllllllll}1 & 2 & 3 & 4 & 5 & 6 & 7 & 8 & 9\end{array}$

Subjects

Figure 12. GRT Fork Able-Bodied Data

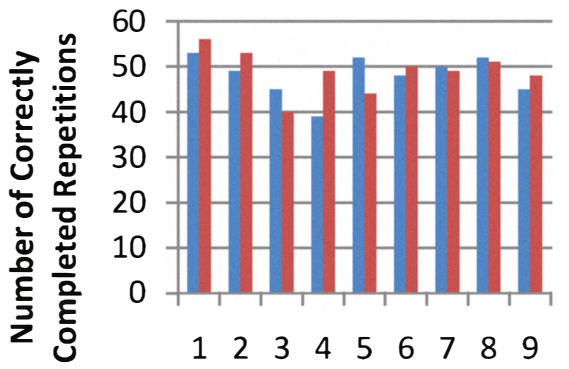

Subjects

Figure 13. GRT Can Able-Bodied Data


Subjects

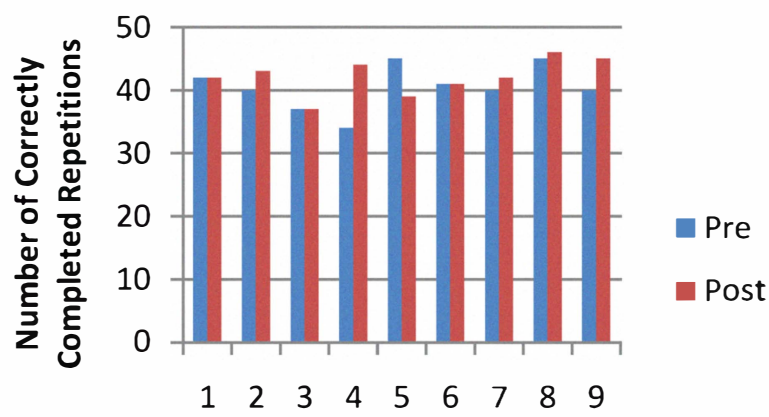

Subjects

Figure 15. GRT Tape Able-Bodied Data

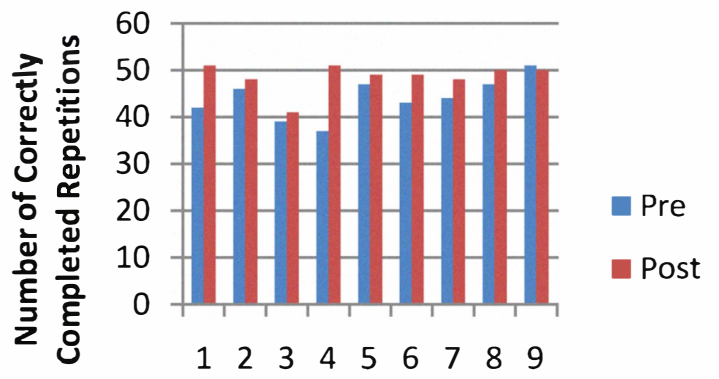

Subjects

Figure 16. GRT Block Able-Bodied Data

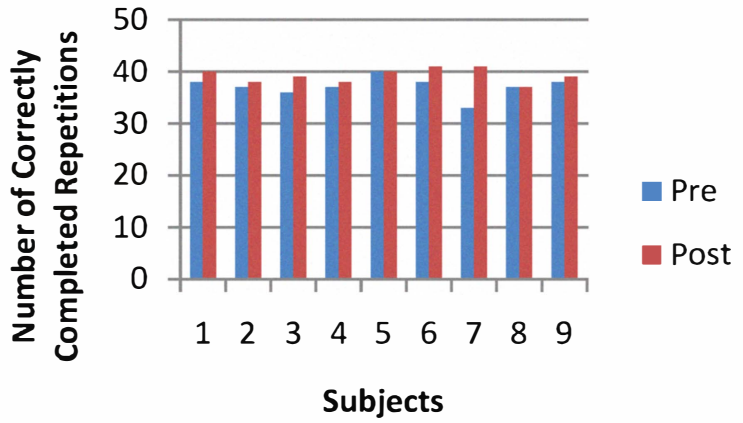

Figure 17. GRT Peg Able-Bodied Data

We did not expect to see any significant change in the ablebodied population from our intervention, and this result is exactly what we observed. However, this data will prove useful as a baseline by which to measure potential change in people with SCI. 


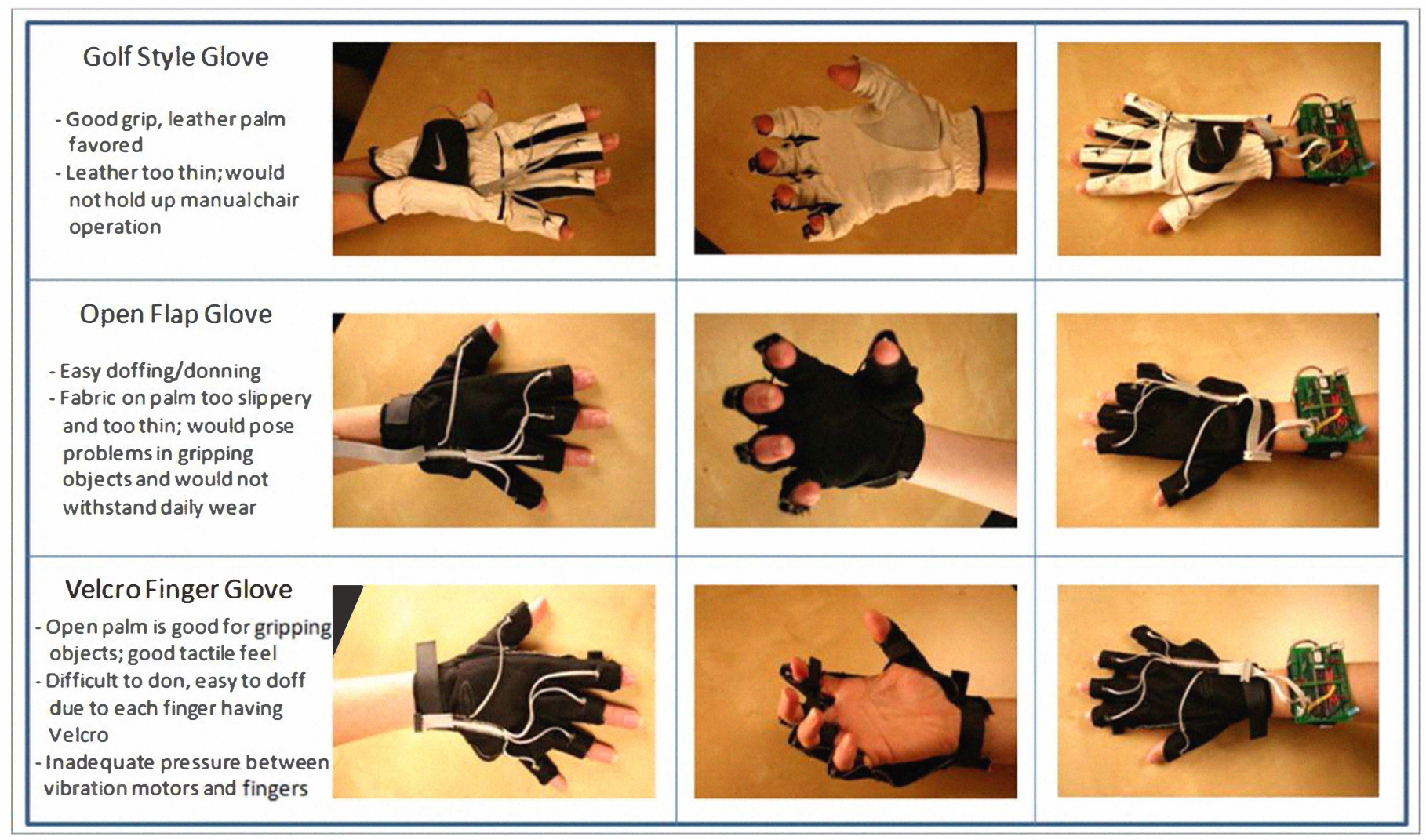

Figure 18. Glove Comparison Chart

\section{Glove Design}

In order to ensure the glove design we use for the full SCI "active practice" study was easy to use for the participants, we designed two new gloves to compare with the original concept glove. We designed the gloves in conjunction with our Industrial Design team.

The Open Flap Glove is a modified glove that has the finger tips cut off and longitudinal slits down both sides. This enables the glove to be opened very wide to assist in donning the glove on "clawed" hands. Additionally, small lateral slits have been cut into each finger above the knuckle to allow for proper motor placement. The wires are contained by a Velcro strap, and the hardware attaches to a Velcro patch near the wrist.

The Velcro Finger Glove is also a modified glove, but the entire ventral side of the glove has been removed and Velcro straps wrap around the user's fingers. Wire management is achieved through two sets of external straps.

We performed a user study to determine which glove design was most suited for regular/everyday use by people with SCI. This type of study was employed to better understand the user experience. The participants in the study were three individuals with tetraplegia who played together on a wheelchair rugby team. The benefit of using these subjects was that they are considerably active with their hands and would put the gloves through rigorous testing. Each participant was given all three glove designs and instructed to wear each design six hours a day for two consecutive days while performing normal daily activities. Following each glove usability session, participants were asked to complete a nineteen question satisfaction survey assessing the performance of both the glove and hardware components. The survey contained both structured and open-ended questions with the intent of obtaining standard measures of functionality for the glove as well as personal experiences that may dictate further design. To emulate a system that interfaces with a Handheld Music Player or a Mobile phone, we preprogrammed the glove to keep generating the finger vibrations for the song "Jingle Bells" every 100 seconds. The Glove, when switched on, will wait for a Bluetooth connection for a minute, if it doesn't find a connection, it starts vibrating as it would when playing "Jingle Bells." Each finger vibrated for 500 milliseconds and paused for another 500 milliseconds before vibrating the next finger. A spectrogram of the vibration motors used is presented in figure 19 below.

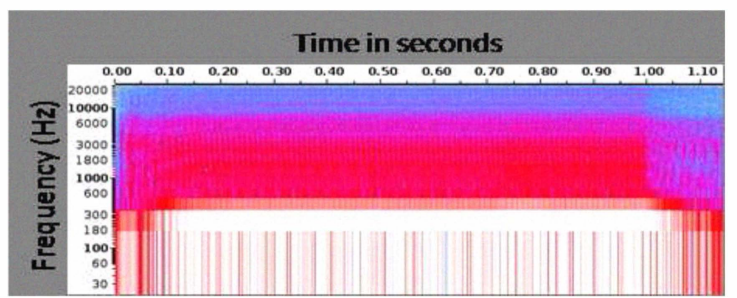

Figure 19. Spectrogram of Vibration Motors used in the MMT System. 
The participants' responses to the surveys have been compiled, categorized by glove design, and are presented in Figure 18 on the preceding page and in text below. Due to the small subject population, it is difficult to make statistical inferences from this data, but the feedback is helpful in making design considerations for future studies.

Glove 1: Golf Style Glove. This was the design of the glove used for the pilot study [3]. The golf style glove design received "satisfied" to "very satisfactory" reviews with respect to comfort, fit, and grip. The participants experienced difficulty donning the glove as they did not have assistance in this domain. The glove itself was not considered irritating, and the weight was only slightly noticeable. However, one user expressed dissatisfaction in glove material as it would easily wear from continual contact with the wheelchair wheels. The glove itself was considered to be of little hindrance to daily activities. The hardware feedback consisted of similar issues pertaining to the other gloves. Exposed wire, hardware size, and hardware slippage were all noticeable and contributed to maneuverability problems and hindered daily activities. The hardware produced good vibration cues with participants being able to distinguish the cues from individual motors. The hardware was also listed as not working reliably during one participant's session as the wire leads to the batteries kept detaching. Another participant described feeling higher intensity in vibrations in the fourth and fifth fingers but was uncertain whether it was due to a hardware issue or hypersensitivity in the fingers.

Glove 2: Open Flap Glove. The open flap glove received mixed reviews and proved to be highly dependent on individual preference and the severity of a participant's tetraplegia. The feedback indicated that the users were dissatisfied with the material selection, as the slick fabric did not afford gripping and thus hindered manual wheelchair handling. Participants also gave the glove dissatisfactory reviews with respect to the durability fabric and the fasteners. User comfort ranged from dissatisfied to very satisfied, and all three users were satisfied with overall fit. The users did not find the glove to be irritating or the weight to be too noticeable. Furthermore, participants stated that putting on and removing the glove posed some to no difficulty as users employed teeth to don, adjust, and secure the glove. As per the hardware comfort and software performance, no feedback was received from one participant due to board failure. The two remaining participants were unhappy with the wire exposure, though the ribbon cable was securely sewn to the glove with some slack left to account for finger bending and extension. The size of the hardware proved to be another point of dissatisfaction with the participants. General bulkiness and an unsecure forearm mount resulted in continual slippage. This contributed to maneuverability difficulties and impedance with daily activities such as hygiene tasks and chair pushing. In two instances, participants indicated the hardware as being irritating to the hand or arm. The performance of the glove was rated well for administering clear, distinguishable vibration cues. However, the overall ratings for hardware reliability varied as the wire leads to the batteries kept detaching in one of the gloves.

Glove 3: Velcro Finger Glove. While the Velcro finger glove did receive mixed reviews by way of comfort, fit, and durability, it was clear from the remaining survey responses and user commentary that this glove was the least preferred of the three designs. All users strongly disagreed that the glove was easy to put on; however, some participants indicated that glove removal was easy. The weight of the glove itself was not considered to be irritating, but the users did state the glove was noticeable when donned with hardware. Two participants indicated that the glove irritated or rubbed the hand/forearm, while all participants considered the hardware to interfere with daily activities. The users responded that vibration motors were easily discernable, but there was some feedback on lower vibration intensity due to shifting of the fingerlets. One user indicated that the hardware performance was insufficient as the wire lead to the battery pack detached.

We also observed some issues that were mentioned by the participants with SCI in reference to using the MMT with a manual wheelchair. Problems encountered included hardware slippage down the arm, entangling with the wheelchair, lack of grip or no grip on the palm of the gloves to provide traction while pushing the wheels of the wheelchair, and finally difficulty putting the glove and hardware assembly on by oneself.

Overall Assessment. No single glove design was the most preferred by all users. Instead, select features of each particular glove design proved to be desirable. After reviewing the feedback, it was clear that one glove design may not be appropriate for accommodating the different levels of tetraplegia. Thus, the severity of the disability as well as particular user needs have to be taken into account when deciding which features to incorporate into a new glove design.

The glove design process is iterative in nature. This first iteration revealed several issues and concerns that had not been known going into this study. Based on these findings, a second iteration of this study will be conducted with refinements to the glove designs based on user feedback. Additional changes would include reducing hardware size, implementing the housing compartment with the working system, mounting the hardware on the wrist rather than the arm, selecting a glove with more tractional material, better wire management (including full stitching to the base of the vibration motors) and exploring the possibility of using conductive thread for optimal wire management.

\section{FUTURE WORK}

Glove refinements will be made based on feedback discussed above and another wearability study will be conducted to find the optimal combination of attributes that accommodates the largest group of users among the SCI 
population. The MMT "active practice" pilot study will be repeated with a larger group of SCI participants to validate the rehabilitative effects observed during the pilot study. Next, "mobile" system gloves will be deployed with a group of persons with SCI to attempt to isolate what is responsible for any rehabilitative effects: the vibration stimulus, the act of playing the piano, the music, or some combination of these. We will also continue the study of the passive haptic learning concept with the MMT system, exploring the effect of long term (daily) wear of the glove with vibration stimulus, to evaluate if there is a greater learning impact than that observed by short term wear versus a control, as examined in the previous study.

\section{CONCLUSION}

MMT looks promising as a form of hand rehabilitation in persons suffering tetraplegia as a result of incomplete SCI. The preliminary work we presented in this paper will enable us to perform this study on persons with SCI to see if it is beneficial to the tetraplegia population at large.

\section{ACKNOWLEDGMENTS}

We wish to thank Kris Walters for granting permission to use his spectrogram image of our vibration motors (figure 19).

\section{REFERENCES}

[1] M.J. Mulcahey, D. Hutchinson, and S. Kozin. "Assessment of upper limb in tetraplegia: considerations in evaluation and outcomes research.," in Journal of Rehabilitation Research \& Development, vol. 44, pp. 91-102, 2007.

[2] N. Yozbatiran, L. Der-Yeghiaian, and S.C. Cramer. "A standardized approach to performing the Action Research Arm Test," in Neurorehabilitation and Neural Repair, vol. 22, 2007.

[3] K. Huang, E. Yi-Luen Do, T. Starner, D. Kohlsdorf, and C. Ahlrichs. "Mobile Music Touch: mobile tactile stimulation for passive learning," in press, CHI 2010.

[4] E. Moberg. "Two Point Discrimination Test. A valuable part of hand surgical rehabilitation, e.g. in tetraplegia," in Scandinavian Journal of Rehabilitative Medicine, vol. 22, pp. 127-134, 1990.

[5] D. Jack, R. Boian, A. Merians, S.V. Adamovich, M. Tremaine, M. Recce, G.C. Burdea, and H. Poizner. "A virtual reality-based exercise program for stroke rehabilitation," in Assets '00: Proceedings of the Fourth International ACM Conference on Assistive Technologies, 2000.

[6] M.M. Dimitrijevic, N. Soroker, and F.E. Pollo. "Mesh glove electrical stimulation," in Science \& Medecine, vol. 3, 1996.

[7] N. Doidge. The Brain that Changes Itself. New York, NY: Viking, 2007.

[8] C.M. Zelazny. "Theraputic instrumental music playing in hand rehabilitation for older adults with osteoarthritis: four case studies," in Journal of Music Therapy, pp. 97-113, 2001.

[9] J.W. Little, B. Goldstein, M. Hammond. "Chapter 5: Spinal Cord Injury Rehabilitation." Rehabilitation of the Injured Combatant. Volume 1. Ed. T.R. Dillingham and P.V. Belandres. Washington DC: Office of the Surgeon General at TMM Publications Institute, pp. 162-205, 1998. 